\title{
La voie Notch
}

Du développement à la régénération du muscle squelettique

> Les muscles du squelette des vertébrés dérivent de structures mésodermiques transitoires: les Alicia Mayeuf, Frédéric Relaix somites. Dans la partie dorsale des somites, le dermomyotome, on trouve des cellules progénitrices capables de contribuer à la formation de l'ensemble des muscles du squelette du tronc et des membres. L'engagement de ces progéniteurs dans le programme de différenciation musculaire est induit par l'activation des facteurs de régulation myogéniques. Les progéniteurs fournissent l'ensemble des cellules myogéniques pendant la croissance de l'embryon et du fœtus et adoptent une position en périphérie des fibres musculaires lors de la période post-natale. Ces cellules quiescentes, appelées cellules satellites, constituent un réservoir de cellules souches musculaires post-natales qui permettent la croissance et la régénération musculaires. Parmi les mécanismes qui contrôlent leur maintien à l'état de progéniteur, on trouve la voie Notch. Cette voie de signalisation est également essentielle à la formation des somites et au développement des muscles du squelette chez l'embryon. Au cours du vieillissement, l'activité de la voie Notch diminue, ce qui participe à la diminution des capacités régénératrices du muscle. Cette voie de signalisation est donc un régulateur majeur de la biologie du muscle. <

Le tissu musculaire squelettique est incroyablement plastique. Cette plasticité ne saurait exister sans la présence de cellules progénitrices, capables à chaque instant de s'engager dans le programme myogénique pour former du muscle. L'établissement et la pérennité de cette réserve de cellules souches sont essentiels au maintien de l'intégrité du tissu musculaire après une lésion ou son endommagement. Une voie de signalisation joue un rôle essentiel dans ces processus : il s'agit de la voie Notch. Cette revue retrace le rôle de la voie

Notch dans le système myogénique et fait le point sur ses fonctions tout au long de la vie du muscle, pendant sa formation, sa croissance, sa régénération et son vieillissement.

\section{Croissance et régénération des muscles}

Parmi les tissus des vertébrés, le muscle du squelette est l'un des plus remarquables. II existe 640 muscles chez l'homme, avec une grande variété de tailles, formes et propriétés physiologiques. Alors que certains muscles participent aux fonctions vitales de l'organisme, comme les muscles respiratoires, d'autres s'adaptent au métabolisme et à l'activité physique de l'organisme, lui permettant ainsi de se nourrir et de se mouvoir afin d'assurer la posture la plus adaptée à sa condition. Ce tissu présente également un développement et une croissance prodigieux joints à une morphogenèse complexe et coordonnée avec les tissus auxquels il s'associe (nerfs, os, vaisseaux sanguins, tendons et tissu connectif). D'un point de vue cellulaire, le tissu musculaire possède de grandes capacités régénératrices. Ses capacités de croissance et de régénération font de lui un organe très plastique. À l'origine de la plasticité musculaire se trouve un réservoir de cellules souches, dont les plus étudiées à ce jour sont les cellules satellites qui, comme leur nom l'indique, se localisent en périphérie des fibres musculaires, sous la lame basale [1]. Ces cellules sont mises en place au cours du développement, lors de la formation des muscles à partir des progéniteurs musculaires embryonnaires [2]. Elles sont présentes dans toutes les masses musculaires, où elles résident dans un état quiescent jusqu'au moment où des signaux viennent les solliciter [3]. Les cellules progénitrices du muscle chez l'embryon et les cellules satellites chez l'adulte contribuent donc à la formation des muscles du squelette, à leur croissance et à leur régénération. II n'est donc pas étonnant de 


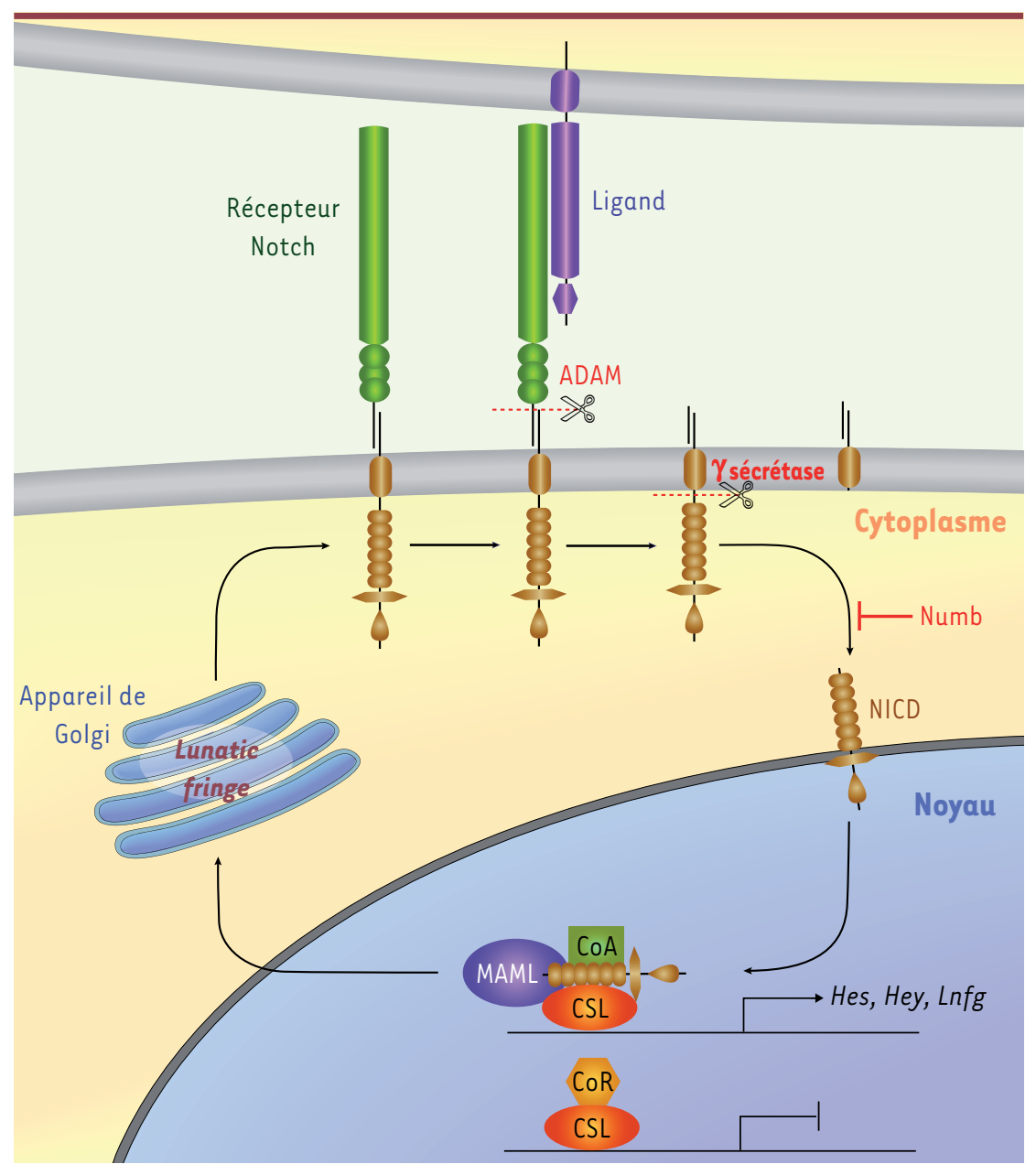

Figure 1. Représentation schématique de la voie de signalisation Notch. Le récepteur Notch se compose de deux domaines: le domaine intracellulaire (jaune) et le domaine extracellulaire (vert), lequel interagit avec les ligands Delta et Jagged (violet). L'interaction du récepteur avec son ligand entraîne le clivage du récepteur par des metalloprotéases de la famille ADAM (a disintegrin and metalloprotease) puis par le complexe $\gamma$-sécrétase, et libère le domaine intracellulaire du récepteur (NICD) dans le cytoplasme de la cellule réceptrice. Ce domaine contient un signal de localisation nucléaire (NLS). Le NICD est transloqué dans le noyau. II interagit avec le complexe CSL associé à des corépresseurs (CoR), ce qui permet le recrutement de coactivateurs transcriptionnels ( $C O A)$ et l'activation de la transcription des gènes cibles de la voie Notch (Hes, Hey, lunatic fringe, etc.). Lunatic fringe est une glycosyltransférase qui modifie de manière post-traductionnelle les récepteurs Notch lors de leur synthèse. Numb est une protéine localisée dans le cytoplasme dont la fonction est d'empêcher la translocation nucléaire du NICD. MAML : mastermind-like transcriptional co-activator. constater que certains mécanismes sont conservés par ces cellules au cours du temps, notamment des mécanismes impliqués dans le contrôle de leur autorenouvellement. C'est par exemple le cas de la voie de signalisation Notch qui joue un rôle crucial à chaque étape de la vie du muscle.

\section{La voie de signalisation Notch}

La voie de signalisation Notch a fait l'objet de nombreuses études dans des modèles et des systèmes variés. Très conservée au cours de l'évolution, cette voie de signalisation est impliquée dans de nombreux mécanismes cellulaires et joue notamment un rôle important dans le contrôle de la différenciation et le choix d'un destin cellulaire [4, 5]. Les premières études réalisées l'ont été chez la drosophile, chez qui il existe un seul récepteur Notch et deux ligands: Delta et Serrate [6]. Chez les vertébrés, il y a eu des duplications au cours de l'évolution, et il existe par exemple quatre récepteurs Notch (Notchl-4), ainsi que cinq ligands (Delta-like 1, 3, 4 et Jagged 1, 2) chez la souris [7]. L'interaction d'un récepteur Notch avec l'un de ses ligands entraîne le clivage de la partie intracellulaire du récepteur, appelé NICD pour Notch intracellular domain (Figure 1). Cette forme clivée constitue la forme active du récepteur Notch. Celle-ci migre dans le noyau où elle interagit avec un complexe transcriptionnel composé du facteur de transcription CSL (CBFl/RBPJ-K/Su(H)/ Lag- $\left.1^{1}\right)$, lequel est déjà positionné sur les séquences régulatrices des gènes cibles de Notch. L'introduction de NICD dans le complexe entraîne la libération des corépresseurs et le recrutement de coactivateurs, permettant ainsi l'activation transcriptionnelle des gènes cibles de la voie. Parmi ceux-ci, des facteurs de transcription à domaine de liaison bHLH (basic helix loop helix) de type répresseur, appartenant aux familles des gènes Hes (hairy/enhancer of split) et Hey, ont été largement caractérisés [8]. L'expression des acteurs de la voie de signalisation Notch, tout comme l'expression de ses gènes cibles, est très dynamique et finement régulée d'un point de vue spatiotemporel. D'autre part, les régulateurs de la voie Notch sont nombreux. Ils se localisent dans différents compartiments cellulaires et

${ }^{1} \mathrm{RBPJ}-\mathrm{K}$ : recombination signal binding protein for immunoglobulin $\kappa$ J region 
agissent à différents niveaux de la voie. Par exemple, parmi ceux-ci, lunatic fringe est une glycosyltransférase, localisée dans l'appareil de Golgi, qui agit sur la voie Notch en modifiant l'affinité des récepteurs pour leurs différents ligands [9].

\section{Notch et la formation des somites}

Avant même l'initiation de la formation des muscles chez l'embryon, la voie Notch joue un rôle crucial. L'ensemble de la musculature axiale et appendiculaire des vertébrés dérive de structures transitoires embryonnaires : les somites. Ces structures épithéliales sont issues d'un mécanisme de segmentation du mésoderme para-axial, contrôlé par une horloge moléculaire [10], dont l'une des aiguilles principales est la voie Notch [11]. L'activation de la voie Notch dans le mésoderme présomitique est périodique. Cette périodicité est régulée par des mécanismes de répression négative qui induisent l'expression cyclique de gènes cibles (parmi lesquels lunatic fringe et Hes-7) (Figure 2A). La fréquence de passage d'un état actif à un état inactif de la voie Notch régule la périodicité de formation des somites, c'est-à-dire la formation d'une paire de somites toutes les deux heures environ chez l'embryon de souris. Deux autres voies de signalisation coordonnent ce mécanisme : il s'agit des voies Wnt et Fgf (fibroblast growth factor) [12]. La plupart de ces données ont été obtenues à partir de modèles génétiques chez qui des acteurs de la voie Notch ont été mutés. Récemment, une étude basée essentiellement sur l'analyse des phénotypes des mutants Hes7, lunatic fringe, RBPJ- $\kappa$ et des doubles mutants Psen $1 / P_{\text {sen }}{ }^{2}$, chez qui l'activité Notch est totalement absente, a montré que la voie Notch est à la fois requise pour la formation des somites et la génération de leur périodicité, mais aussi que l'ensemble des autres voies de signalisation impliquées dans ce mécanisme sont interconnectées à la voie Notch, faisant de celle-ci un pilier central de la somitogenèse [13].

\section{Notch et la formation des muscles du squelette}

La voie Notch joue donc un rôle essentiel dans la somitogenèse. Mais qu'en est-il un peu plus tard, au cours de la myogenèse embryonnaire, fœtale et adulte? Les premières démonstrations de l'implication de cette voie ont été obtenues grâce à des approches d'embryologie expérimentale et de culture cellulaire. Les résultats ont rapidement indiqué l'importance de cette signalisation dans l'ensemble des étapes de la formation et de la régulation du muscle squelettique.

Lors du développement précoce chez les vertébrés, les somites ont tout d'abord une structure épithéliale, puis ils se compartimentent et s'allongent selon l'axe dorsoventral. Le dermomyotome est situé dans la partie dorsale du somite où résident des cellules épithéliales exprimant Pax3 : un facteur de transcription à homéoboîte et à domaine paired [14] (Figure 2B). Les cellules Pax3+ sont des progéniteurs multipotents capables de participer à la formation de l'ensemble des muscles du squelette du tronc et des membres, mais aussi au derme du dos, à certaines cellules musculaires lisses, endothéliales, et au tissu adipeux brun [15]. L'importance de la voie Notch dans ce choix d'un destin

${ }^{2}$ Psen 1 : préséniline. Les présénilines 1 et 2 sont deux protéines homologues, composant catalytique $d$ complexe gamma-sécrétase responsable du clivage du récepteur Notch (Figure 1). cellulaire a récemment été soulignée chez l'embryon de poulet, où la voie Notch promeut la différenciation des cellules progénitrices en cellules du muscle lisse plutôt qu'en cellules du muscle squelettique [16].

Lorsqu'une cellule progénitrice s'engage dans le programme de différenciation musculaire squelettique, l'expression des facteurs de régulation myogéniques MyoD, Myf5, Mrf4 et myogénine est non seulement activée, mais est essentielle à la détermination et à la différenciation musculaires [2]. De nombreuses études ont démontré que la voie de signalisation Notch est impliquée dans la régulation de l'entrée des cellules dans ce programme. Par exemple, dans des expériences réalisées chez l'embryon de poulet, la voie Notch inhibe la différenciation myogénique et permet le maintien des cellules progénitrices dans un état indifférencié $[17,18]$. II a été proposé que cet effet inhibiteur passe par l'activation de l'expression de Hesl. Ce facteur de transcription est en effet capable de réprimer l'expression de MyoD [19-21]. Toutefois, certaines études ont porté sur les mécanismes moléculaires associés à ce contrôle de la différenciation myogénique. Les résultats ont montré que, bien que la voie canonique RBPJ- $\kappa$ soit clairement impliquée dans ce phénomène, il existe probablement d'autres voies qui restent méconnues en aval du couple ligand/récepteur [22, 23].

Puisque la voie Notch joue un rôle crucial dès les étapes précoces du développement embryonnaire, lors de la somitogenèse, son inactivation entraîne des effets dramatiques. Par conséquent, la mise au point de stratégies d'inactivation conditionnelle chez la souris a été essentielle pour étudier l'implication de la voie Notch dans le système myogénique aux stades plus tardifs du développement embryonnaire. Ces approches permettent en effet d'agir de manière plus ciblée sur les cellules progénitrices du muscle et de façon contrôlée dans le temps. Deux études y ont eu recours qui utilisent deux approches différentes chez la souris. La première était fondée sur l'analyse phénotypique d'embryons hétéroalléliques, possédant un allèle nul et un allèle hypomorphe pour le gène Deltal ${ }^{3}$ [24]. La seconde étude repose sur l'utilisation d'une approche conditionnelle, via le système Cre/lox [25] où le gène codant le facteur de transcription RBPJ- $\kappa$ a été inactivé spécifiquement dans les progéniteurs musculaires [26]. Ces deux approches complémentaires ont mis en évidence l'importance de la voie Notch dans la formation des muscles embryonnaires et fœtaux chez la souris. Elles ont confirmé l'implication de celle-ci dans le contrôle de la différenciation myogénique, c'est-à-dire

${ }^{3}$ Les embryons qui possèdent deux allèles nuls pour ce gène n'étant pas viable au-delà de 11 jours de développement embryonnaire. 
le maintien des progéniteurs musculaires dans un état indifférencié. En effet, lorsque la voie Notch est affectée, la différenciation des cellules progénitrices devient excessive, ce qui entraîne l'épuisement et la perte irréversible du réservoir de progéniteurs disponibles. À des stades embryonnaires avancés $(\varepsilon 14,5-\varepsilon 16,5)$, les mutants conditionnels RBPJ- $\kappa$ et les mutants Deltal se caractérisent par des masses musculaires atrophiées. Ainsi, la voie de signalisation Notch, non seulement est à l'origine de la formation des somites, mais elle joue également un rôle primordial dans la myogenèse embryonnaire et fœtale. Et pourtant, la fonction de la voie Notch dans le système myogénique ne s'arrête pas là.

\section{Notch et la régénération musculaire}

Les cellules satellites, formées à partir des progéniteurs embryonnaires, expriment le facteur de transcription Pax7, ainsi que son homologue Pax3 dans certains muscles $[27,28]$. Ces cellules souches du muscle du squelette adulte demeurent dans un état quiescent, en périphérie des fibres musculaires, et sont activées lors de la croissance musculaire post-natale, lors d'exercices ou, plus généralement, lors d'endommagement des fibres musculaires qu'elles remplacent [29]. Une fois activées, elles prolifèrent avant de se différencier. Un réservoir de cellules souches est cependant toujours conservé afin que ce processus puisse être réitéré en cas de nouvelle demande [30]. Plusieurs études ont révélé l'importance de la voie Notch dans le phénomène de régénération musculaire. Les cellules satellites expriment en effet le récepteur Notchl. Lorsque des fibres musculaires sont mises en culture, ce qui est assimilé à un dommage musculaire, la voie Notch devient active suite à l'apparition de l'expression du ligand Deltal à la surface des cellules satellites, des myofibres et des cellules interstitielles du muscle. L'expression de Deltal en réponse à une lésion musculaire serait induite par des signaux qui ne sont, à ce jour, pas identifiés. L'activation de la voie Notch par Deltal dans les cellules satellites permet leur sortie de quiescence et active leur prolifération. Le nombre de progéniteurs musculaires est ainsi amplifié avant que ceux-ci ne se différencient et ne forment de nouvelles fibres musculaires (Figure 2C). L'activation constitutive de Notchl dans les cellules satellites augmente donc leur prolifération et diminue leur différenciation. Au contraire, l'inhibition de la voie Notch par la protéine Numb entraîne la sortie des cellules satellites du cycle cellulaire et induit leur différenciation [31]. Les effets de la voie Notch décrits lors de la myogenèse embryonnaire sont par conséquent réitérés chez l'adulte. Le rôle de cette voie de signalisation dans le système myogénique est donc de maintenir le réservoir de cellules souches en inhibant leur différenciation et en favorisant leur autorenouvellement afin de maintenir l'homéostasie du tissu musculaire. II est toutefois important de noter que l'ensemble de ces études se sont focalisées sur le récepteur Notchl. Une étude récente vient cependant de mettre en évidence que les fonctions des différents récepteurs Notch lors de la régénération musculaire ne sont pas équivalentes. En effet, le récepteur Notch3 agit comme un inhibiteur de la prolifération et de l'autorenouvellement des cellules satellites lors de dommages musculaires par l'intermédiaire du répresseur de l'activité Notch, Nrap [32]. Les fonctions opposées de ces deux récepteurs lors de la régénération permettent ainsi le maintien de l'homéostasie du muscle après sa détérioration.

\section{Notch au cours du vieillissement musculaire}

Le muscle est un tissu qui se renouvelle peu en conditions normales, mais qui possède, comme nous venons de l'exposer, de grandes capacités régénératrices. Toutefois, ce potentiel se réduit peu à peu au cours du vieillissement, conduisant à une atrophie musculaire progressive [33]. Puisque la voie Notch est un acteur majeur de la régénération musculaire, l'hypothèse selon laquelle elle pourrait également être impliquée lors du vieillissement musculaire a été émise. Même s'il n'est pas clairement établi que le réservoir de cellules satellites s'appauvrit au cours du vieillissement, une chose est sûre: le potentiel myogénique des cellules satellites se réduit, et l'activité de la voie Notch diminue de manière concomitante [34] (Figure 2C). En effet, à la suite d'une lésion musculaire, l'expression de Deltal est induite dans les cellules satellites et les fibres musculaires, mais de façon moins importante dans les muscles d'individus âgés que ceux d'individus jeunes. L'expression du récepteur Notchl n'est quant à elle pas modifiée selon l'âge de l'individu. En revanche, l'activation du récepteur par son ligand est elle aussi réduite dans les cellules satellites d'individus âgés. D'autre part, lorsque l'activation de la signalisation Notch est bloquée dans les cellules satellites de muscles jeunes, la régénération musculaire est inhibée. Lorsqu'on force l'activation de la voie Notch dans les cellules satellites de muscles âgés, ces derniers régénèrent mieux. II existe, par conséquent, une corrélation directe entre la régénération musculaire liée à l'âge et l'activation de la voie Notch dans les cellules satellites. II a donc été proposé que les capacités régénératrices des muscles âgés ne sont pas perdues de manière irréversible, mais qu'elles sont probablement sous le contrôle de signaux environnementaux qui varient au cours du temps [35]. Un argument en ce sens est venu d'expériences de parabiose hétérochroniques où ont été connectés les appareils circulatoires de deux souris : une souris jeune et une âgée. Ces expériences ont révélé une augmentation de la régénération musculaire chez les souris âgées (après une lésion induite) et une diminution de celle-ci chez les souris jeunes [35]. Ces résultats démontrent que la régénération musculaire est liée à un ou des facteurs systémiques (qui ne sont pas identifiés à ce jour) qui contrôlent l'activation de l'expression de Deltal, l'activation de la voie Notch et la régénération, et dont la présence s'amenuise au cours du vieillissement. En parallèle, chez les individus âgés, il existe certainement des facteurs défavorables à l'activation des cellules satellites et donc à la régénération.

En conclusion, l'environnement cellulaire est un élément-clé de la régénération du muscle puisque c’est lui 


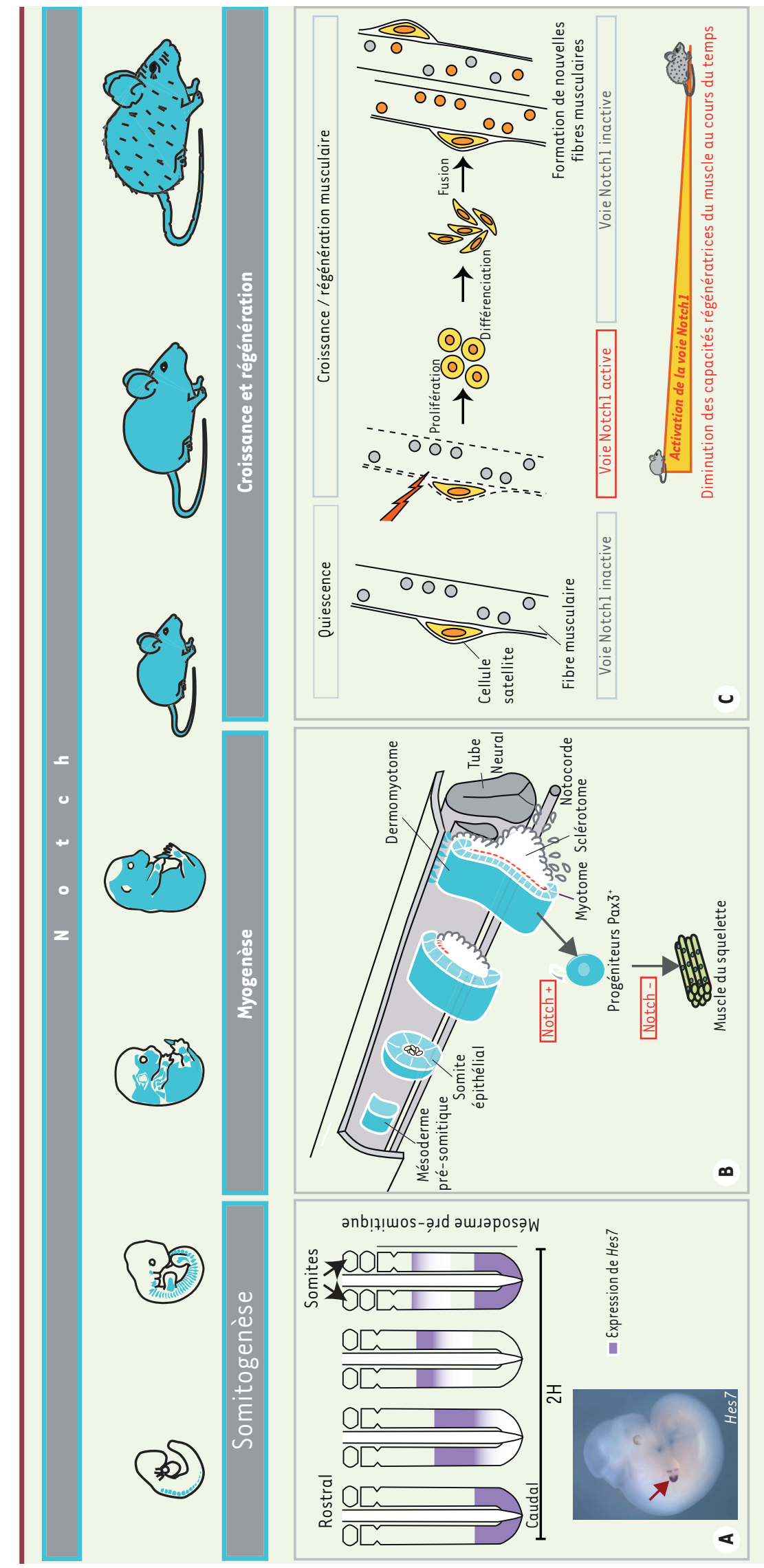

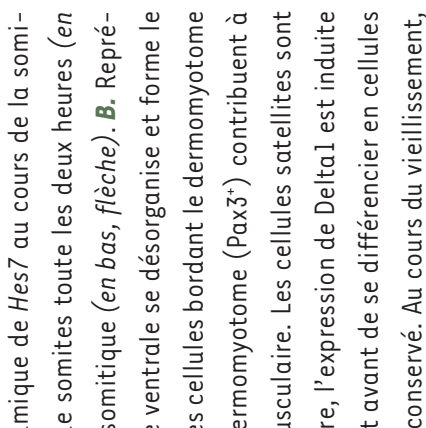

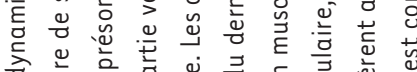



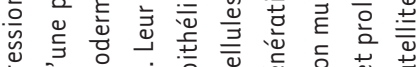

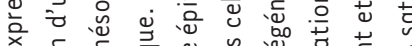
区

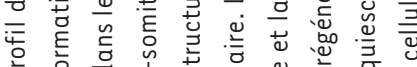

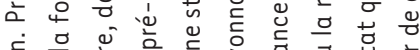

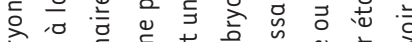

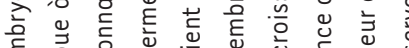

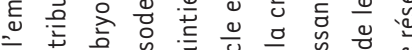

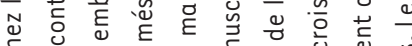

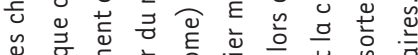

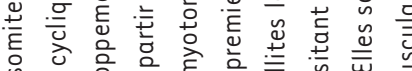
ज ᄃ 巡 음 담 焉 言 $<\dot{ن}$ ن

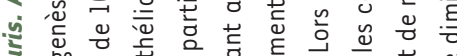
वํ. 들

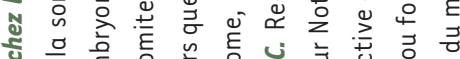

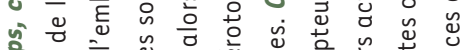
है

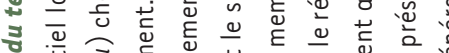

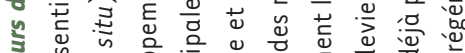
\& $\widetilde{U} \cong$ 응

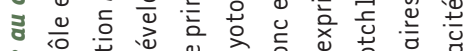

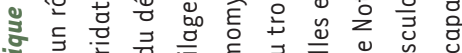
势

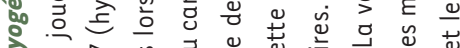

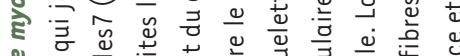


पे

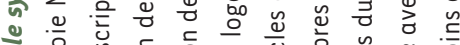
y

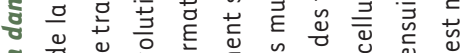
苛

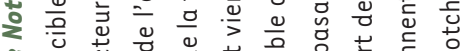

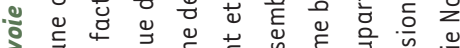

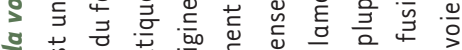

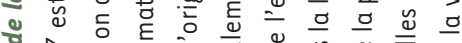
ะ

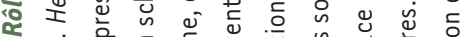
ن

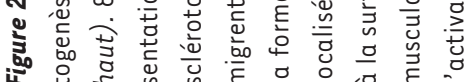

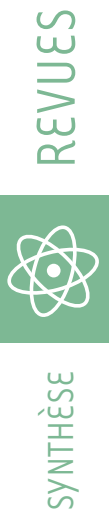


qui contrôle l'homéostasie cellulaire de ce tissu. Des perturbations de cet environnement affectent ses capacités régénératrices et représentent un mécanisme physiologique retrouvé au cours du vieillissement. Un des enjeux actuels est d'identifier ces facteurs solubles, afin de mieux comprendre les mécanismes sous-jacents de la régénération musculaire et de les mettre à profit en thérapie. $\diamond$

\section{SUMMARY}

Notch pathway: from development to regeneration of skeletal muscle In vertebrates, skeletal muscle is derived from mesodermal structures called somites. Myogenic progenitor cells that form skeletal muscles of the trunk and limbs are derived from the dermomyotome, the dorsal region of the somite. These cells enter the myogenic program by activating a set of four myogenic regulatory factors. During embryonic and fetal growth, muscle progenitor cells provide the source for muscle growth. Around birth, the muscle progenitor enters quiescence, and adopts a satellite cell position on muscle fibers, providing a pool of adult muscle stem cells. They are essential for the growth and regeneration of muscles. Among the mechanisms that control the maintenance of satellite cells properties, the Notch pathway plays a crucial role. In facts, this pathway is implicated from the early steps of somitogenesis and the development of skeletal muscles in the embryo. Furthermore, during ageing, Notch activity decreases which results in decreased muscle regeneration. Thus, the Notch pathway is a key regulator of muscle plasticity. $\diamond$

\section{REMERCIEMENTS}

Nous remercions Didier Rocancourt pour son aide dans l'élaboration des figures. A.M. remercie le ministère de l'Enseignement et de la recherche, l'UPMC, la Société Française de Myologie, et F.R. remercie I'Inserm, I'UPMC et l'AFM pour leur soutien financier.

\section{CONFLIT D'INTÉRÊTS}

Les auteurs déclarent n'avoir aucun conflit d'intérêts concernant les données publiées dans cet article.

\section{RÉFÉRENCES}

1. Mauro A. Satellite cell of skeletal muscle fibers. J Biophys Biochem Cytol $1961 ; 9: 493-5$.

2. Lagha M, Rocancourt D, Relaix F. Origine du muscle squelettique : rôles de Pax3/Pax7. Med Sci (Paris) $2005 ; 21: 801-3$

3. Zammit PS, Partridge TA, Yablonka-Reuveni Z. The skeletal muscle satellite cell : the stem cell that came in from the cold. J Histochem Cytochem $2006 ; 54: 1177-91$.

4. Lai $\varepsilon C$. Notch signaling : control of cell communication and cell fate. Development 2004 ; $131: 965-73$.

5. Borggrefe T, Oswald F. The Notch signaling pathway : transcriptional regulation at Notch target genes. Cell Mol Life Sci $2009 ; 66$ : 1631-46.

6. Brou C, Logeat F. Endocytose et voie de signalisation Notch. Med Sci (Paris) $2006 ; 22: 685-8$

7. Kopan R, llagan MX. The canonical Notch signaling pathway : unfolding the activation mechanism. Cell $2009 ; 137: 216-33$

8. Bray SJ. Notch signalling : a simple pathway becomes complex. Nat Rev Mol Cell Biol 2006 ; $7: 678-89$.

9. Yang LT, Nichols JT, Yao C, et al. Fringe glycosyltransferases differentially modulate Notch proteolysis induced by Deltal and Jaggedl. Mol Biol Cell $2005 ; 16$ : 927-42.

10. Freitas $C$, Rodrigues $S$, Charrier JP, et al. Horloge moléculaire et segmentation des vertébrés : qui fait quoi ? Med Sci (Paris) $2002 ; 18: 883-7$.
11. Dequeant ML, Pourquie 0. Segmental patterning of the vertebrate embryonic axis. Nat Rev Genet $2008 ; 9: 370-82$.

12. Ozbudak $E M$, Pourquie 0 . The vertebrate segmentation clock : the tip of the iceberg. Curr Opin Genet Dev $2008 ; 18: 317-23$

13. Ferjentsik Z, Hayashi S, Dale JK, et al. Notch is a critical component of the mouse somitogenesis oscillator and is essential for the formation of the somites. PLoS genetics $2009 ; 5$ : e1000662.

14. Buckingham M, Relaix F. The role of Pax genes in the development of tissues and organs: Pax3 and Pax7 regulate muscle progenitor cell functions. Annual review of cell and developmental biology $2007 ; 23: 645-73$.

15. Buckingham $M$, Vincent SD. Distinct and dynamic myogenic populations in the vertebrate embryo. Curr Opin Genet Dev 2009 ; 19: 444-53.

16. Ben-Yair R, Kalcheim C. Notch and bone morphogenetic protein differentially act on dermomyotome cells to generate endothelium, smooth, and striated muscle. J Cell Biol 2008 ; $180: 607-18$.

17. Delfini MC, Hirsinger $\varepsilon$, Pourquie 0 , Duprez D. Delta l-activated notch inhibits muscle differentiation without affecting Myf5 and Pax3 expression in chick limb myogenesis. Development 2000 ; 127 : 5213-24.

18. Hirsinger $\varepsilon$, Malapert $P$, Dubrulle J, et al. Notch signalling acts in postmitotic avian myogenic cells to control MyoD activation. Development 2001 ; $128: 107-16$.

19. Kopan R, Nye JS, Weintraub $H$. The intracellular domain of mouse Notch : a constitutively activated repressor of myogenesis directed at the basic helixloop-helix region of MyoD. Development 1994 ; 120 : 2385-96.

20. Jarriault $\mathrm{S}$, Brou $\mathrm{C}$, Logeat $\mathrm{F}$, et al. Signalling downstream of activated mammalian Notch. Nature $1995 ; 377: 355-8$

21. Kuroda K, Tani S, Tamura K, et al. Delta-induced Notch signaling mediated by RBP-J inhibits MyoD expression and myogenesis. J Biol Chem 1999; 274 $7238-44$

22. Buas MF, Kabak S, Kadesch T. Inhibition of myogenesis by Notch : evidence for multiple pathways. J Cell Physiol 2009; $218: 84-93$

23. Shawber C, Nofziger D, Hsieh JJ, et al. Notch signaling inhibits muscle cell differentiation through a CBFl-independent pathway. Development 1996 ; $122: 3765-73$.

24. Schuster-Gossler K, Cordes R, Gossler A. Premature myogenic differentiation and depletion of progenitor cells cause severe muscle hypotrophy in Deltal mutants. Proc Natl Acad Sci USA 2007 ; $104: 537-42$

25. Sauer B. Inducible gene targeting in mice using the Cre/lox system. Methods $1998 ; 14: 381-92$

26. Vasyutina $\varepsilon$, Lenhard DC, Wende $H$, et al. RBP-J (Rbpsuh) is essential to maintain muscle progenitor cells and to generate satellite cells. Proc Natl Acad Sci USA 2007 ; 104 : 4443-8.

27. Seale $P$, Sabourin LA, Girgis-Gabardo A, et al. Pax 7 is required for the specification of myogenic satellite cells. Cell $2000 ; 102$ : 777-86.

28. Relaix F, Rocancourt D, Mansouri A, Buckingham M. A Pax3/Pax7-dependent population of skeletal muscle progenitor cells. Nature $2005 ; 435: 948-53$.

29. Tedesco FS, Dellavalle A, Diaz-Manera J, et al. Repairing skeletal muscle : regenerative potential of skeletal muscle stem cells. J Clin Invest 2010 $120: 11-9$.

30. Buckingham M, Montarras D. Skeletal muscle stem cells. Curr Opin Genet Dev $2008 ; 18: 330-6$

31. Conboy IM, Rando TA. The regulation of Notch signaling controls satellite cell activation and cell fate determination in postnatal myogenesis. Dev Cell $2002 ; 3: 397-409$

32. Kitamoto T, Hanaoka K. Notch 3 null mutation in mice causes muscle hyperplasia by repetitive muscle regeneration. Stem Cells $2010 ; 28: 2205-16$.

33. Conboy IM, Rando TA. Aging, stem cells and tissue regeneration : lessons from muscle. Cell Cycle $2005 ; 4$ : 407-10

34. Conboy IM, Conboy MJ, Smythe GM, Rando TA. Notch-mediated restoration of regenerative potential to aged muscle. Science $2003 ; 302$ : 1575-7.

35. Conboy IM, Conboy MJ, Wagers AJ, et al. Rejuvenation of aged progenitor cells by exposure to a young systemic environment. Nature $2005 ; 433: 760-4$.

\section{TIRÉS À PART}

F. Relaix 